\title{
Solving A Multi-Objective Reactive Power MARKET CLEARING MODEL USING NSGA-II
}

\author{
Ashish Saini ${ }^{1}$ and Amit Saraswat ${ }^{2}$ \\ Department of Electrical Engg., Faculty of Engineering, \\ Dayalbagh Educational Institute, India-282110, Uttar Pradesh, India. \\ ${ }^{1}$ ashish7119@gmail.com, and ${ }^{2}$ amitsaras@gmail.com
}

\begin{abstract}
This paper presents an application of elitist non-dominated sorting genetic algorithm (NSGA-II) for solving a multi-objective reactive power market clearing (MO-RPMC) model. In this MO-RPMC model, two objective functions such as total payment function (TPF) for reactive power support from generators/synchronous condensers and voltage stability enhancement index (VSEI) are optimized simultaneously while satisfying various system equality and inequality constraints in competitive electricity markets which forms a complex mixed integer nonlinear optimization problem with binary variables. The proposed NSGA-II based MO-RPMC model is tested on standard IEEE 24 bus reliability test system. The results obtained in NSGA-II based MO-RPMC model are also compared with the results obtained in real coded genetic algorithm (RCGA) based single-objective RPMC models.
\end{abstract}

\section{KEYWORDS}

Reactive power market clearing, competitive electricity markets, NSGA-II, multi-objective optimization, pareto-optimal front

\section{INTRODUCTION}

Almost all real world optimization problems involve optimizing (i.e. whether minimization or maximization or combinations of both) the multiple objective functions simultaneously. In fact, these objective functions are non-commensurable and often conflicting objectives. Multiobjective optimization with such conflicting objective functions gives rise to a set of optimal solutions, instead of one optimal solution [1]. In general, a common multi-objective optimization problem may be formulated [1] as follows:

$$
\begin{aligned}
& \text { Minimize } \quad f_{i}(x) \quad \forall i=1, \ldots \ldots \ldots, N_{o b j} \\
& \text { subject to }:\left\{\begin{array}{ll}
g_{j}(x)=0 & j=1, \ldots \ldots \ldots, M, \\
h_{k}(x) \leq 0 & k=1, \ldots \ldots \ldots, K,
\end{array}\right\}
\end{aligned}
$$

Where $f_{i}(x)$ is the $i^{\text {th }}$ objective function, $x$ is a decision vector that represents a solution, and $N_{o b j}$ is the number of objective functions, $M$ and $N$ are number of system equality and inequality constraints respectively. For a multi-objective optimization problem, any two solutions $x_{1}$ and $x_{2}$ can have one of two possibilities- one dominates the other or none dominates the other. In a DOI : $10.5121 /$ ijait.2012.2304 
minimization problem, without loss of generality, a solution $\mathrm{x}_{1}$ dominates $\mathrm{x}_{2}$, if and only if, the following two conditions are satisfied:

$$
\begin{aligned}
& \forall i=\left\{1, \ldots \ldots \ldots, N_{o b j}\right\}: f_{i}\left(x_{1}\right) \leq f_{i}\left(x_{2}\right) \\
& \exists j=\left\{1, \ldots \ldots \ldots, N_{o b j}\right\}: f_{j}\left(x_{1}\right)<f_{j}\left(x_{2}\right)
\end{aligned}
$$

If any of the above conditions is violated, the solution $x_{1}$ does not dominate the solution $x_{2}$. If $x_{1}$ dominates the solution $x_{2}$, then $x_{1}$ is called the non-dominated solution within the set $\left\{x_{1}, x_{2}\right\}$. The solutions that are non-dominated within the entire search space are denoted as Pareto-optimal and constitute the Pareto-optimal set or Pareto-optimal front [1]. In recent years, several evolutionary multi-objective solution techniques such as Strength Pareto Evolutionary Algorithm (SPEA) [2], fuzzy adaptive particle swarm optimization (FAPSO) [3], a seekers optimization algorithm (SOA) [4], a modified non-dominated sorting genetic algorithm (MNSGA-II) [5] are applied to reactive power optimization problems such as optimal reactive power dispatch ORPD [2-5], congestion management [6] and RPMC [7].

In competitive electricity markets engineers view the reactive power management problem from two angles - technical and as well as economical [8-9]. A market model process to manage reactive services by independent transmission operators are presented in [10] and uses a piecewise linear representation of the capability curve of each generator for computing reactive power cost curves. Zhong et al. [11-12] developed a competitive market for reactive power and raised many important issues of reactive power management. In [11], a two-step approach for reactive power procurement is proposed. This work is extended in [12], where a uniform price auction model was proposed to competitively determine the prices for different components of reactive power services namely: availability, operation and opportunity. Market clearing was achieved by simultaneously considering minimization of payment, total system losses, and deviations from contracted transactions using compromise programming approach, which is based on single objective optimization framework.

In present paper, the multi-objective reactive power market clearing (MO-RPMC) problem is formulated as a mixed integer nonlinear multi-objective optimization problem with binary variables which includes two objectives such as total payment function (TPF) for rective power support from generators/syncronus condensers [12] and voltage stability enhancement index (VSEI) [13-14]. A non-dominated sorting genetic algorithm (NSGA-II) as developed in reference [15] is applied for solving the MO-RPMC problem and tested on IEEE 24 bus reliability test system. The result obtained in proposed NSGA-II based MO-RPMC model is compared with a result obtained in single-objective (SO-RPMC) model using real coded genetic algorithm (RCGA) [16].

\section{Proposed MO-RPMC Model: Problem Formulation}

The proposed MO-RPMC model involves a complex, highly constraints multi-objective optimization problem described as follows:

\subsection{Objective Functions}

The first objective function is to minimize the total payment function $(T P F)$ for reactive power support services provided by generators and synchronous condensers in order to settle the reactive power market. The principle of highest priced offer selected determining the market price is 
applied with additional system constraints. As in reference [12], The TPF for reactive supports may be formulated as follows:

$$
F_{1}=T P F=\sum_{i \in N_{P V}}\left(\rho_{0} \cdot W_{0, i}-\rho_{1} \cdot W_{1, i} \cdot Q_{G 1, i}+\rho_{2} \cdot W_{2, i} \cdot Q_{G 2, i}+\rho_{2} \cdot W_{3, i} \cdot Q_{G A, i}+\frac{1}{2} \rho_{3} \cdot W_{3, i} \cdot Q_{G 3, i}^{2}\right)
$$

Reactive power output from $i^{\text {th }}$ provider is classified into three components $Q_{G 1, i}, Q_{G 2, i}$ or $Q_{G 3, i}$ that represent the regions $\left(Q_{\text {Gmini, }}, 0\right),\left(Q_{G \text { bass }, i}, Q_{G A, i}\right)$ and $\left(Q_{G A, i}, Q_{G B, i}\right)$, respectively. Accordingly, only one of the binary variables $W_{1}, W_{2}$ and $W_{3}$ can be selected. In (1), $\rho_{0}$ is the uniform availability price and $\rho_{1}$ and $\rho_{2}$ are the uniform cost of loss prices, whereas $\rho_{3}$ is the uniform opportunity price. If a provider is selected, $W_{0}$ will be one, and it will receive the availability price, irrespective of its reactive power output.

The second objective is to minimize a voltage stability enhancement index (VSEI) also known as L-index [13] in order to incorporate the voltage stability improvement in RPMC scheme. It is a static voltage stability measure of power system, computed based on normal load flow solution as presented in [14] solution. Its value may be defined as follows:

$$
F_{2}=V S E I=L-\text { index }=\max \left\{L_{j}=\left|1-\sum_{i=1}^{N_{p V}} F_{j i} \frac{V_{i}}{V_{j}}\right|, j \in N_{P Q}\right\}
$$

All the terms within the sigma of (6) are complex quantities. The values $F_{j i}$ are obtained from the Y-bus matrix as below in (7).

$$
\left[\begin{array}{c}
I_{G} \\
I_{L}
\end{array}\right]=\left[\begin{array}{ll}
Y_{G G} & Y_{G L} \\
Y_{L G} & Y_{L L}
\end{array}\right]\left[\begin{array}{c}
V_{G} \\
V_{L}
\end{array}\right]
$$

Where $\left[I_{G}\right],\left[I_{L}\right]$ and $\left[V_{G}\right],\left[V_{L}\right]$ represents the complex currents and bus voltages respectively; whereas $\left[Y_{G G}\right],\left[Y_{G L}\right],\left[Y_{L G}\right]$ and $\left[Y_{L L}\right]$ are corresponding portions of network Y-bus matrix.

Rearranging (7), we obtain

$$
\left[\begin{array}{c}
V_{L} \\
I_{G}
\end{array}\right]=\left[\begin{array}{ll}
Z_{L L} & F_{L G} \\
R_{G L} & Y_{G G}
\end{array}\right]\left[\begin{array}{c}
I_{L} \\
V_{G}
\end{array}\right]
$$

$$
\text { Where } F_{L G}=-\left[Y_{L L}\right]^{-1}\left[Y_{L G}\right]
$$

The value of $L$-index lies between 0 and 1 [14]. An $L$-index value less than 1 (voltage collapse point) and close to 0 (no load point) indicates a system state i.e. system voltage stability margin. For a given network, as the load/generation increases, the voltage magnitude and angles change, and for near maximum power transfer condition, the voltage stability index $L_{j}$ values for load buses tends close to 1 , indicating that the system is close to voltage collapse.

\subsection{System Operating Constraints}

The following system operating equality and inequality constraints are satisfied: 


\subsubsection{Load flow equality constraints}

$$
\begin{aligned}
& P_{G, i}-P_{D, i}-V_{i} \sum_{j \in N_{i}} V_{j}\left(G_{i j} \cos \theta_{i j}+B_{i j} \sin \theta_{i j}\right)=0 ; \quad i \in N_{B} \\
& Q_{G, i}-Q_{D, i}-V_{i} \sum_{j \in N_{i}} V_{j}\left(G_{i j} \sin \theta_{i j}+B_{i j} \cos \theta_{i j}\right)=0 ; \quad i \in N_{B}
\end{aligned}
$$

where $N_{B}$ is the number of buses; $P_{G}$ and $Q_{G}$ are the generator real and reactive power, respectively; $V_{j}$ is the voltage magnitude at $j^{\text {th }}$ bus; $P_{D}$ and $Q_{D}$ are the load real and reactive power, respectively; $G_{i j}$ and $B_{i j}$ are the transfer conductance and susceptance between bus $i$ and bus $j$, respectively.

\subsubsection{Reactive power relational constraints and limits}

The reactive power offer regions as explained in section 2, a set of governing algebraic relations is required to ensure appropriate allocation as given in (12)-(16).

$$
\begin{aligned}
& Q_{G i}=Q_{G 1, i}+Q_{G 2, i}+Q_{G 3, i} \\
& W_{1, i} \cdot Q_{G \min , i} \leq Q_{G 1, i} \leq W_{1, i} \cdot Q_{G b a s e, i} \\
& W_{2, i} \cdot Q_{G b a s e, i} \leq Q_{G 2, i} \leq W_{2 i} \cdot Q_{G A, i} \\
& W_{3, i} \cdot Q_{G A, i} \leq Q_{G 3, i} \leq W_{3, i} \cdot Q_{G B, i} \\
& W_{1, i}+W_{2, i}+W_{3, i} \leq 1
\end{aligned}
$$

\subsubsection{Constraints determining the market prices}

The market prices $\left(\rho_{0}, \rho_{1}, \rho_{2}\right.$ and $\left.\rho_{3}\right)$ are determined separately for each component of reactive power. The constraints as mentioned in (17)-(20) ensure that the market price, for a given set of offers, is the highest priced offer accepted:

$$
\begin{aligned}
& W_{0, i}=W_{1, i}+W_{2, i}+W_{3, i}, \quad \forall i \in N_{P V} \\
& W_{0, i} \cdot a_{0, i} \leq \rho_{0} \\
& W_{1, i} \cdot m_{1, i} \leq \rho_{1} \\
& \left(W_{2, i}+W_{3, i}\right) \cdot m_{2, i} \leq \rho_{2} \\
& W_{3, i} \cdot m_{3, i} \leq \rho_{3}
\end{aligned}
$$

\subsubsection{Reactive power provision limits}

$$
\begin{aligned}
& Q_{G \min , i} \leq Q_{G, i} \leq Q_{G \max , i} ; \quad i \in N_{P V} \\
& Q_{C \min , i} \leq Q_{C, i} \leq Q_{C \max , i} ; \quad i \in N_{C}
\end{aligned}
$$


Where $Q_{G}, Q_{G \min }$ and $Q_{G \max }$ are reactive power output of generator, its minimum and maximum limits respectively. Similarly, $Q_{C}, Q_{C \min }$ and $Q_{C \max }$ are reactive power from capacitor/inductor banks, its minimum and maximum limits respectively. $N_{P V}$ and $N_{C}$ are total number of generator buses and capacitor/inductor banks respectively.

\subsubsection{Reactive power capability limits of generators}

$$
Q_{G, i}^{\mathrm{limit}} \leq\left\{\begin{array}{c}
\sqrt{\left(V_{t, i} I_{a, i}\right)^{2}-P_{G, i}^{2}} \quad \text { if } P_{G, i} \geq P_{G R, i} \\
\sqrt{\left(\frac{V_{t, i} E_{a f, i}}{X_{s, i}}\right)^{2}-P_{G, i}^{2}}-\frac{V_{t, i}^{2}}{X_{s, i}} \quad \text { if } P_{G, i} \leq P_{G R, i}
\end{array}\right.
$$

\subsubsection{Bus voltage limits}

$V_{i}^{\min } \leq V_{i} \leq V_{i}^{\max } ; \quad \forall i \in N_{P Q}$

$\left|V_{i}\right|=$ Constant; $\forall i \in N_{P V}$

Where $V_{i}, V_{i}^{\min }$ and $V_{i}^{\max }$ are bus voltages, minimum and maximum bus voltage limits respectively. $N_{P Q}$ is the total number of load buses.

\subsubsection{Security constraints}

$$
\begin{aligned}
& S_{l} \leq S_{l}^{\max } ; \quad l \in N_{L} \\
& P_{G \text { min,Slack }} \leq P_{G, \text { Slack }} \leq P_{G \text { max } \text { Slack }}
\end{aligned}
$$

Where $S_{l}$ is line loadings and $S_{l}^{\max }$ maximum permissible loading limit of $l^{\text {th }}$ transmission line. $N_{L}$ is the total number of transmission lines. $P_{G \text { min,Slack }}$ and $P_{G \max , \text { Slack }}$ are the minimum and maximum limits of real power output of slack bus.

\subsubsection{Transformer taps setting constraints}

$T_{k}^{\min } \leq T_{k} \leq T_{k}^{\max } ; \quad k \in N_{T}$

Where $N_{T}$ is total number of transformers.

\subsection{Generalized augmented objective function}

In this paper, a static penalty function approach is used to handle the inequality constraint violations. Infeasible solutions are penalized, by applying a constant penalty to those solutions, which violate feasibility in any way. Thus, the penalty functions corresponding to voltage violations at all load busses, reactive power violations at all generator busses, real power violations at slack bus and power flow violations at all transmission lines $\left(\lambda_{V L, i}, \lambda_{Q G, j}, \lambda_{P G, S l a c k}\right.$ and $\left.\lambda_{S, l}\right)$ are included in objective function as follows: 


$$
\begin{aligned}
F_{a u g, n}= & F_{n}+\sum_{i \in N_{P Q}} \lambda_{V L, i}\left(V_{i}-V_{i}^{\mathrm{lim}}\right)^{2}+\sum_{j \in N_{G}} \lambda_{Q G, j}\left(Q_{G, j}-Q_{G, j}^{\mathrm{lim}}\right)^{2}+\sum_{k \in N_{G, S \text { Salak }}} \lambda_{P G, \text { Slack }}\left(P_{G, k}-P_{G, k}^{\mathrm{lim}}\right)^{2} \\
& +\sum_{l \in N_{L}} \lambda_{S, l}\left(S_{l}-S_{l}^{\mathrm{lim}}\right)^{2} ; \quad \forall n=1: N_{o b j}
\end{aligned}
$$

Where $F_{n}$ are the $n^{\text {th }}$ objective function values and the dependent variable's limiting values may be considered as:

$$
\begin{array}{rlr}
V_{i}^{\lim } & =\left\{\begin{array}{lll}
V_{i}^{\max } ; & \text { if } & V_{i}>V_{i}^{\max } \\
V_{i}^{\min } ; & \text { if } & V_{i}<V_{i}^{\min }
\end{array} ;\right. & \forall i=1: N_{P Q} \\
Q_{G, j}^{\lim } & =\left\{\begin{array}{lll}
Q_{G \max , j} ; & \text { if } & Q_{G, j}>\mathrm{Q}_{G \max , j} \\
Q_{G \min , j} ; & \text { if } & Q_{G, j}<\mathrm{Q}_{G \min , j}
\end{array} ;\right. & \forall j=1: N_{P V} \\
S_{l}^{\lim }=\left\{\begin{array}{lll}
S_{l}^{\max } ; & \text { if } & S_{l}>S_{l}^{\max } \\
S_{l}^{\min } ; & \text { if } & S_{l}<S_{l}^{\min }
\end{array} ;\right. & \forall l=1: N_{L} \\
P_{G, k}^{\lim }=\left\{\begin{array}{lll}
P_{G \max , k} ; & \text { if } & P_{G, k}>P_{G \max , k} ; \\
P_{G \min , k} ; & \text { if } & P_{G, k}<P_{G \min , k}
\end{array}\right. & \forall k=1: N_{G, \text { Slack }}
\end{array}
$$

\section{NSGA-II FOR SOLVING MO-RPMC MODEL}

An elitist non-dominated sorting genetic algorithm (NSGA-II) is implemented for solving complex multi-objective problems like MO-RPMC model. The detail flowchart of proposed algorithmic is shown in Figure 1. Some of its significant features are discussed as below:

Initialization: This requires input of power system data (i.e. bus data, generator data and transmission line data in as specific format) and various parameters of NSGA-II such as population size (popsize), maximum numbers of generations (maxgen), number of control variables, system constraints limits, initial crossover probability $\left(P_{C}\right)$, initial mutation probability $\left(P_{M}\right)$ etc.

Generation of Initial population: Each individual in initial population is a randomly generating string of real valued numbers corresponding to all control variable within their respective minimum and maximum ranges and its validity is checked by running load flow analysis to satisfied both the equality constraints as given in (10)-(11).

Non-Domination Sorting: The generated initial population is sorted on the basis on nondomination sorting algorithm proposed in reference [1] and [15].

For producing the new population for next generation, three evolutionary operators such as tournament selection, SBX crossover and polynomial mutation [15] are applied to parent population. 


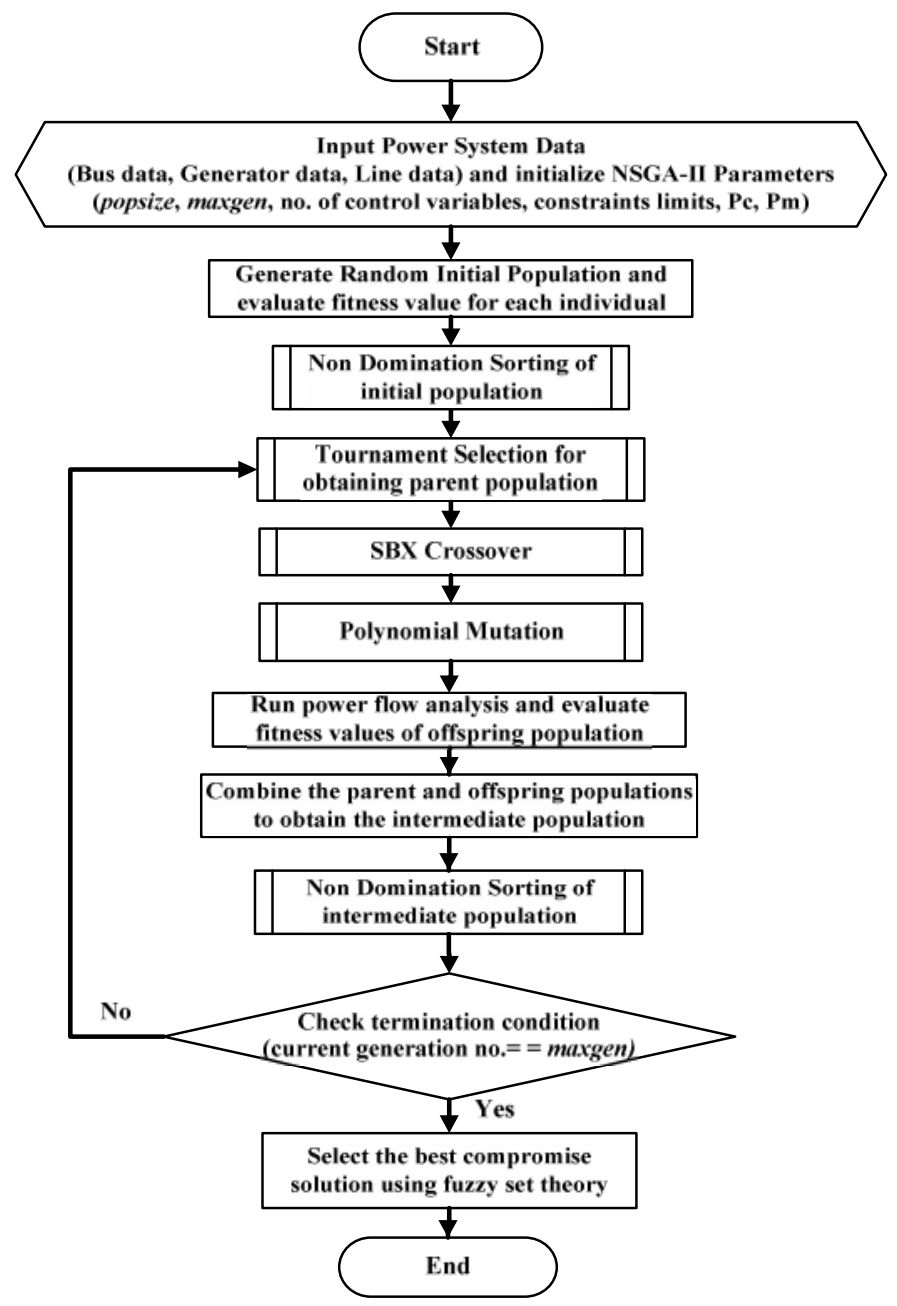

Figure 1. Flowchart of proposed NSGA-II for solving MO-RPMC model

Criterion to prepare population for next generation: After the execution of above evolutionary operators, offspring population is checked to prepare new population for next generation by going through following procedural steps, till the termination condition is not satisfied:

Step 1: Run the Newton-Raphson based load flow analysis on each individual in offspring population;

Step 2: Combine parent and offspring populations to obtain intermediate population;

Step 3: Perform the non-domination sorting algorithm on intermediate population;

Step 4: Remove the worse individuals to maintain the new population size constant. Here the new population for next generation is prepared;

Step 5: Check the termination condition of NSGA-II. i.e. if the current generation number is equal to maxgen, terminate the generation process otherwise go to next generation.

Step 8: Select the Best compromise solution.

Best compromise solution: Upon having the Pareto-optimal set of non-dominated solutions using NSGA-II, an approach proposed in [17] selects one solution to the decision maker as the best compromise solution as used in [2]. This approach suggests that due to imprecise nature of the 
decision maker's judgment, the $i^{\text {th }}$ objective function $F_{i}$ is represented by a membership function $\mu_{i}$ defined as in [2]:

$\mu_{i}=\left\{\begin{array}{cc}1 & F_{i} \leq F_{i}^{\min } \\ \frac{F_{i}^{\max }-F_{i}}{F_{i}^{\max }-F_{i}^{\min }} & F_{i}^{\min }<F_{i}<F_{i}^{\max } \\ 0 & F_{i} \geq F_{i}^{\max }\end{array}\right.$

Where $F_{i}{ }^{\min }$ and $F_{i}{ }^{\max }$ are the minimum and maximum values of the $i^{\text {th }}$ objective function among all non-dominated solutions, respectively. For each $j^{\text {th }}$ non-dominated solution, the normalized membership function $\mu^{j}$ is calculated as:

$\mu^{j}=\frac{\sum_{i=1}^{N_{\text {obj }}} \mu_{i}^{j}}{\sum_{j=1}^{N_{\text {dom }}} \sum_{i=1}^{N_{\text {obj }}} \mu_{i}^{j}}$

Where $N_{d o m}$ is the number of non-dominated solutions. The best compromise solution is that having the maximum value of $\mu^{j}$.

\section{Simulation RESUlts}

The effectiveness of the proposed NSGA-II based MO-RPMC model is demonstrated on the IEEE 24 bus Reliability Test System (IEEE 24 RTS) [18]. The single line diagram of IEEE 24 RTS is shown in Fig. 1. The power system consists of 32 synchronous generators, 1 synchronous condenser (located at bus 14), and 17 constant-power type loads. The system total active and reactive loads are $2850 \mathrm{MW}$ and $580 \mathrm{MVAr}$, respectively. All the simulations are carried out in MATLAB 7.0 programming environment on Pentium IV, $2.26 \mathrm{GHz}, 2.0 \mathrm{~GB}$ RAM computer system.

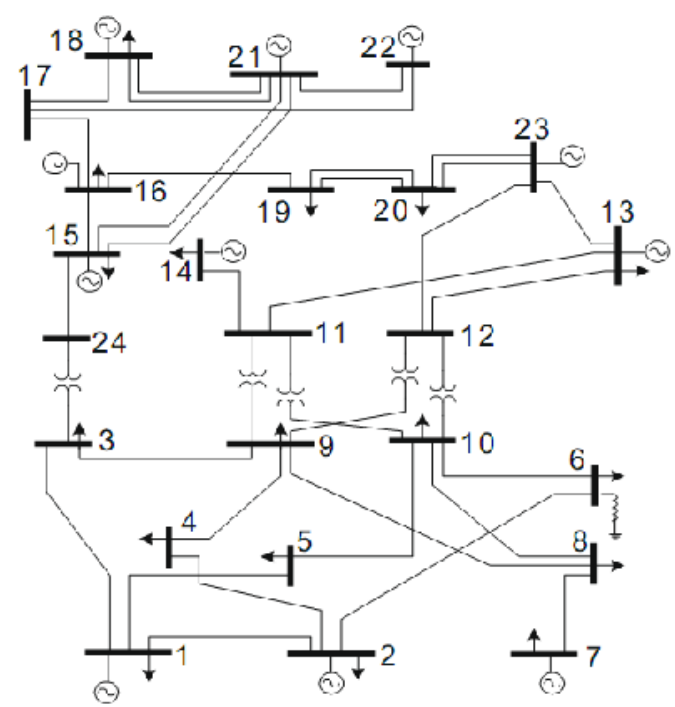

Figure 2. Single line diagram of IEEE 24 bus Reliability Test System 
In this simulation for IEEE 24 RTS, the various system control variables are such as eleven generator bus voltage magnitudes, five transformer tap settings, and one bus shunt admittances. Therefore, the search space has 17 dimensions. The lower and upper limits of all bus voltages are 0.95 p.u. and 1.05 p.u., respectively. The lower and upper limits of all transformer tap settings are 0.9 p.u. and 1.1 p.u, respectively. In order to carry out the RPMC simulations in competitive electricity market environment, the ISO needs the following information from the reactive power providers:

Offer prices: The ISO is supposed to receive four components of the reactive power offer prices $\left(a_{0}^{i, u}, m_{1}^{i, u}, m_{2}^{i, u}\right.$ and $m_{3}^{i, u}$ ), directly from the participants of the reactive power market. In this examination, a uniform random number generator is used to simulate the offer prices of generators as given in reference [19]. It can be observed that the synchronous condenser, is participated in the reactive power market with its opportunity $\operatorname{cost}\left(m_{3}^{i, u}\right)$ equal to zero.

Generator's reactive power capability data: Each participant of the reactive power market (i.e. each generating units) is also required to submit the information regarding its reactive power capability diagram i.e. $Q_{\text {Base }}, Q_{A}$ and $Q_{B}$. In present case study, the assumptions are followed as in references [12] and [19] i.e $Q_{\text {Base }}=0.10 \times Q_{\max }, Q_{A}$ is limited either by the field or the armature heating limit, as per operating condition, and $Q_{B}=1.5 \times Q_{A}$.

Table 1. Case summary of the RPMC simulations

\begin{tabular}{|c|c|c|c|}
\hline Case No. & Optimization Frame Work & Objective Function & Solving Algorithm \\
\hline Case 1 & Single Objective & TPF & RCGA [16] \\
\hline Case 2 & Single Objective & VSEI & RCGA [16] \\
\hline Case 3 & Multi Objective & TPF \& VSEI & NSGA-II \\
\hline
\end{tabular}

Table 2. Specifications of optimization algorithms

\begin{tabular}{|l|l|l|}
\hline Algorithm Parameters & RCGA & NSGA-II \\
\hline length of variables & 17 & 17 \\
\hline Population size $($ Popsize $)$ & 200 & 200 \\
\hline Selection operator & Roulette wheel & Tournament \\
\hline Crossover operator & BLX- $\alpha$ crossover & SBX crossover \\
\hline Mutation Operator & PCA mutation & Polynomial \\
\hline Crossover probability $\left(P_{C}\right)$ & 0.95 & 0.95 \\
\hline Mutation probability $\left(P_{M}\right)$ & 0.015 & 0.015 \\
\hline Maximum generation $($ maxgen $)$ & 500 & 500 \\
\hline
\end{tabular}

In order to maintain the consistency and relevance for comparison, the whole RPMC simulation is divided into three different cases such as case 1,2 and 3 . The summary of all three test cases are given in Table 1. In first two cases, the RPMC problem is treated as single objective optimization problem i.e. only TPF in case 1 and VSEI in case 2 are considered as single objectives for RPMC optimization. In single objective framework, the real coded genetic algorithm (RCGA) as developed by the authors in reference [16] is used to optimize an appropriate objective function while satisfying all the equality (10-11) and inequality constraints (12)-(29). The case 3 is considered as purely a multi-objective model, namely MO-RPMC model, both the objective functions i.e. TPF and VSEI are optimized simultaneously using NAGA-II. For all the three cases, the similar parameters for both the optimization algorithms are taken as: the number of 
maximum iterations $($ max_iterations $=500)$, population size $($ popsize $=200)$ and penalty factors $\left(\lambda_{V L i}=100, \lambda_{Q G j}=50, \lambda_{\text {PGSlack }}=50\right.$ and $\left.\lambda_{S l}=50\right)$. The detailed specifications of RCGA and NSGAII are summarized in Table 2.

Table 3. Comparison of output results from the RPMC simulations

\begin{tabular}{|c|c|c|c|}
\hline Test Case & Case 1 & Case 2 & Case 3 \\
\hline TPF & $\mathbf{3 8 5 . 9 9}$ & 554.96 & $\mathbf{5 0 6 . 3 7}$ \\
\hline VSEI & 0.1842 & $\mathbf{0 . 1 6 0 1}$ & $\mathbf{0 . 1 6 5 8}$ \\
\hline \multicolumn{4}{|c|}{ Uniform Market Clearing Prices (UMCPs) } \\
\hline $\boldsymbol{\rho}_{\mathbf{0}}$ & 0.96 & 0.96 & 0.96 \\
\hline $\boldsymbol{\rho}_{\mathbf{1}}$ & 0 & 0 & 0 \\
\hline $\boldsymbol{\rho}_{\mathbf{2}}$ & 0.86 & 0.86 & 0.86 \\
\hline $\boldsymbol{\rho}_{\mathbf{3}}$ & 0 & 0.46 & 0.35 \\
\hline
\end{tabular}

The optimization results obtained in all three test cases are summarized in Table 3. The bold values in Table 3, represents the optimized objective function values obtained from the RPMC simulations. In single objective RPMC optimization framework, the optimized solutions are such that (385.99 $\$$ and 0.1842$)$ when only TPF is minimized in case 1 , and (554.96 \$ and $\mathbf{0 . 1 6 0 1})$ when only VSEI is minimized in case 2 (see Table 3 ). In cases 3 of multi-objective optimization RPMC framework, the best compromised solutions is selected as (506.37 $\$$ and $\mathbf{0 . 1 7 2 4})$ after optimization using NSGA-II. The uniform market clearing prices i.e. $\operatorname{UMCPs}\left(\rho_{0}, \rho_{1}, \rho_{2}\right.$ and $\left.\rho_{3}\right)$ for reactive power market obtained after execution of RMPC program in all four cases are also mentioned in Table 3 . The best pareto-optimal front and best compromised solution obtained from proposed MO-RPMC model using NSGA-II is shown in Figure 3.

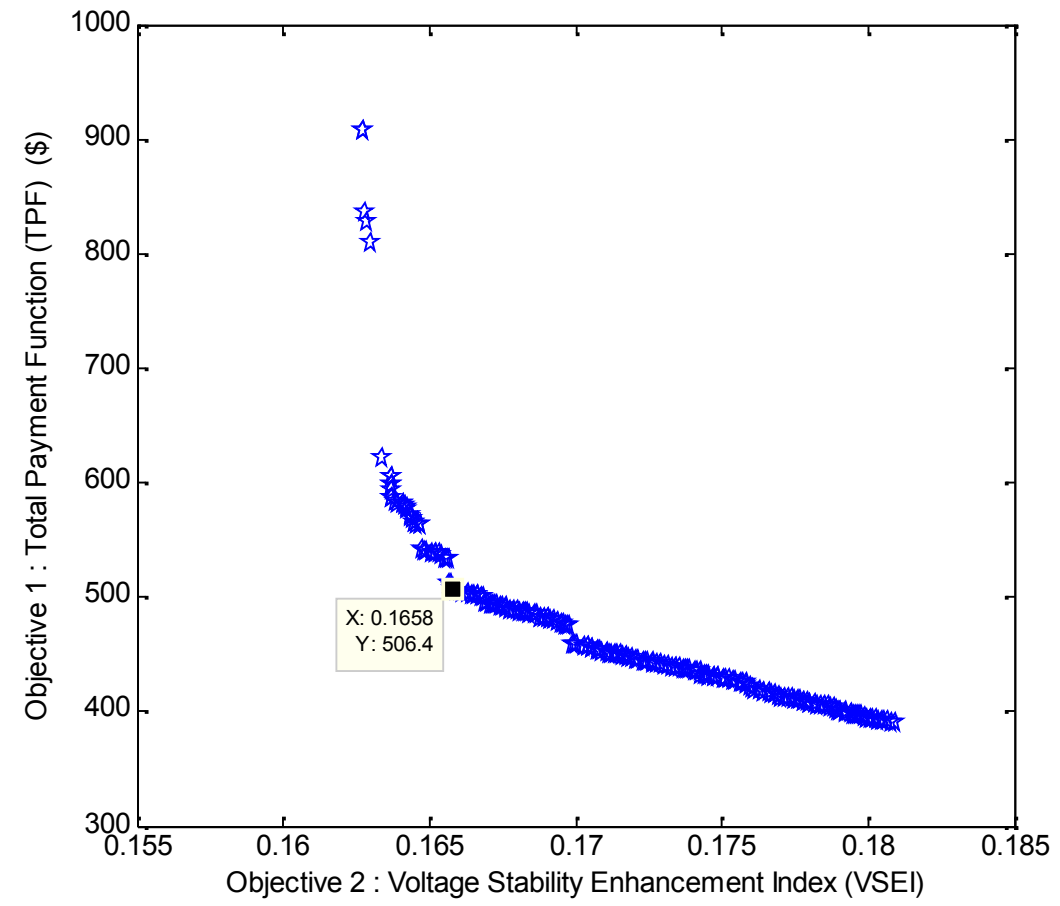

Figure 3. Best pareto-optimal front obtained from multi-objective RPMC model using NSGA-II 
International Journal of Advanced Information Technology (IJAIT) Vol. 2, No.3, June 2012

Table 4. Output control variables obtained from the RPMC simulations

\begin{tabular}{|c|c|c|c|c|}
\hline \multicolumn{5}{|c|}{ Generator bus voltages (p.u.) } \\
\hline Bus No. & ID & Case 1 & Case 2 & Case 3 \\
\hline 1 & $\mathbf{V}_{\mathbf{G 1}}$ & 1.0223 & 1.0494 & 1.0428 \\
\hline 2 & $\mathbf{V}_{\mathrm{G} 2}$ & 1.0234 & 1.0499 & 1.0439 \\
\hline 7 & $\mathbf{V}_{\mathrm{G} 7}$ & 1.0499 & 1.0500 & 1.0489 \\
\hline 13 & $V_{\text {G13 }}$ & 1.0356 & 1.0488 & 1.0254 \\
\hline 14 & $V_{\text {G14 }}$ & 1.0082 & 1.0310 & 1.0053 \\
\hline 15 & $\mathbf{V}_{\mathrm{G} 15}$ & 1.0022 & 1.0186 & 0.9942 \\
\hline 16 & $\mathbf{V}_{\mathrm{G16}}$ & 1.0093 & 1.0255 & 1.0033 \\
\hline 18 & $V_{\text {G18 }}$ & 1.0233 & 1.0289 & 1.0204 \\
\hline 21 & $V_{\text {G21 }}$ & 1.0236 & 1.0348 & 1.0190 \\
\hline 22 & $V_{G_{22}}$ & 1.0419 & 1.0488 & 1.0374 \\
\hline 23 & $V_{\text {G23 }}$ & 1.0408 & 1.0500 & 1.0293 \\
\hline \multicolumn{5}{|c|}{ Transformer tap settings (p.u.) } \\
\hline Bus No. & ID & Case 1 & Case 2 & Case 3 \\
\hline $3-24$ & $\mathbf{T}_{3-24}$ & 1.03 & 1.07 & 1.10 \\
\hline $9-11$ & $\mathbf{T}_{9-11}$ & 1.06 & 1.06 & 1.03 \\
\hline $9-12$ & $\mathbf{T}_{9-12}$ & 1.02 & 1.05 & 1.07 \\
\hline 10-11 & $T_{10-11}$ & 0.98 & 0.94 & 0.95 \\
\hline 10-12 & $T_{10-12}$ & 0.98 & 0.95 & 1.00 \\
\hline \multicolumn{5}{|c|}{ Shunt Capacitances (MVA-r) } \\
\hline Bus No. & ID & Case 1 & Case 2 & Case 3 \\
\hline 6 & $\overline{Q_{\mathrm{C} 6}}$ & -83.86 & -165.52 & -158.35 \\
\hline
\end{tabular}

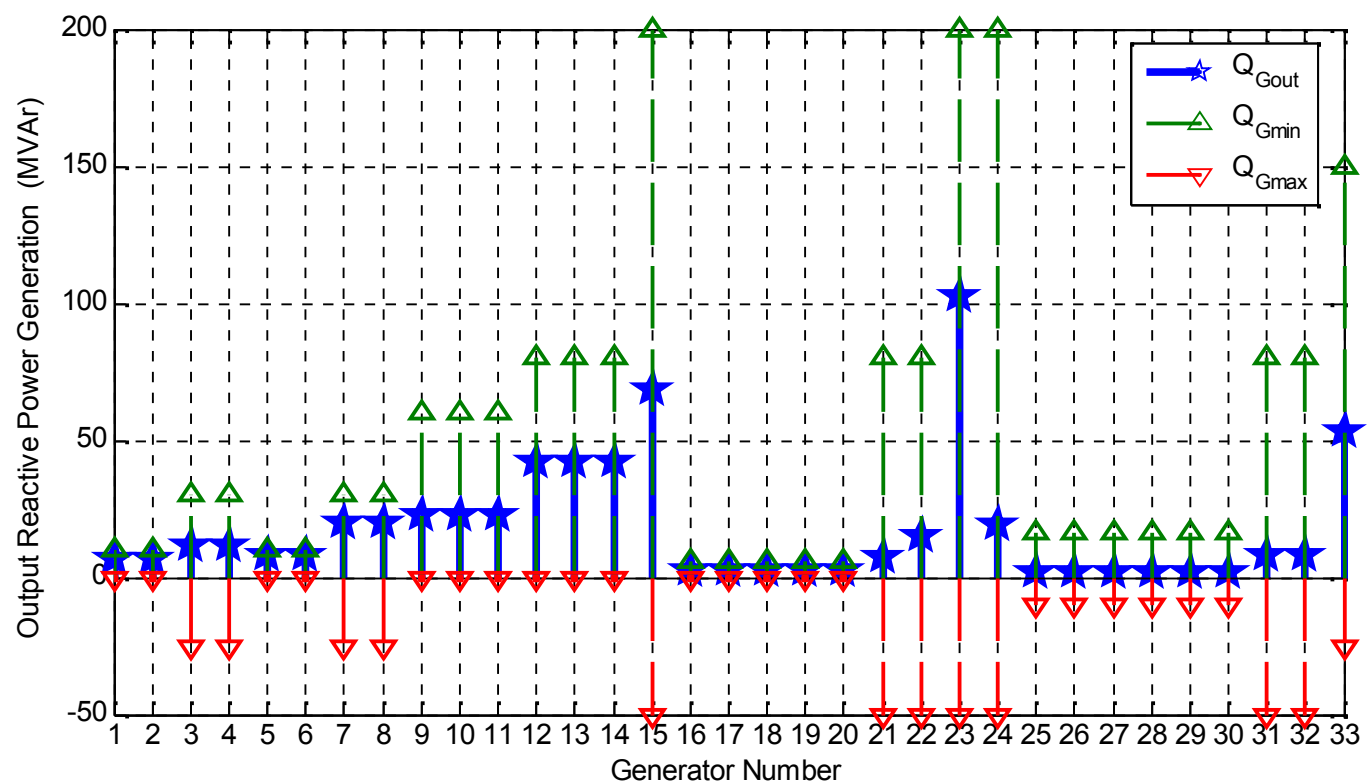

Figure 4. Generators reactive power schedule obtained from NSGA-II based MO-RPMC model 
International Journal of Advanced Information Technology (IJAIT) Vol. 2, No.3, June 2012

Table 4. Output reactive power generation schedules and their payments for all generators obtained from RPMC simulations

\begin{tabular}{|c|c|c|c|c|c|c|c|}
\hline \multirow[b]{2}{*}{$\begin{array}{l}\text { Bus } \\
\text { No. }\end{array}$} & \multirow[b]{2}{*}{$\begin{array}{l}\text { Unit } \\
\text { No. }\end{array}$} & \multicolumn{2}{|c|}{ Case 1} & \multicolumn{2}{|c|}{ Case 2} & \multicolumn{2}{|c|}{ Case 3} \\
\hline & & $\begin{array}{c}\mathbf{Q}_{\mathrm{G}} \\
\text { (MVAr) }\end{array}$ & $\begin{array}{c}\text { Payment } \\
\text { (\$) }\end{array}$ & $\begin{array}{c}\mathbf{Q}_{\mathbf{G}} \\
\text { (MVAr) }\end{array}$ & $\begin{array}{c}\text { Payment } \\
\text { (\$) }\end{array}$ & $\begin{array}{c}\mathbf{Q}_{\mathrm{G}} \\
(\mathrm{MVAr})\end{array}$ & $\begin{array}{c}\text { Payment } \\
\text { (\$) }\end{array}$ \\
\hline \multirow{4}{*}{1} & 1 & 4.99 & 5.25 & 7.39 & 19.26 & 6.64 & 6.67 \\
\hline & 2 & 4.99 & 5.25 & 7.39 & 19.26 & 6.64 & 6.67 \\
\hline & 3 & 2.42 & 0.00 & 15.66 & 14.43 & 11.51 & 10.86 \\
\hline & 4 & 2.42 & 0.00 & 15.66 & 14.43 & 11.51 & 10.86 \\
\hline \multirow{4}{*}{2} & 1 & 5.06 & 5.32 & 7.90 & 21.06 & 8.18 & 18.39 \\
\hline & 2 & 5.06 & 5.32 & 7.90 & 21.06 & 8.18 & 18.39 \\
\hline & 3 & 2.85 & 0.00 & 18.48 & 16.85 & 19.97 & 18.13 \\
\hline & 4 & 2.85 & 0.00 & 18.48 & 16.85 & 19.97 & 18.13 \\
\hline \multirow{3}{*}{7} & 1 & 21.20 & 19.20 & 21.20 & 19.19 & 22.58 & 20.38 \\
\hline & 2 & 21.20 & 19.20 & 21.20 & 19.19 & 22.58 & 20.38 \\
\hline & 3 & 21.20 & 19.20 & 21.20 & 19.19 & 22.58 & 20.38 \\
\hline \multirow{3}{*}{13} & 1 & 41.16 & 36.36 & 41.79 & 36.90 & 42.27 & 37.31 \\
\hline & 2 & 41.16 & 36.36 & 41.79 & 36.90 & 42.27 & 37.31 \\
\hline & 3 & 41.16 & 36.36 & 41.79 & 36.90 & 42.27 & 37.31 \\
\hline $14^{\mathrm{a}}$ & 1 & 75.92 & 66.25 & 96.81 & 84.21 & 68.44 & 59.81 \\
\hline \multirow{6}{*}{15} & 1 & 2.65 & 3.23 & 3.86 & 4.28 & 2.63 & 3.22 \\
\hline & 2 & 2.65 & 3.23 & 3.86 & 4.28 & 2.63 & 3.22 \\
\hline & 3 & 2.65 & 3.23 & 3.86 & 4.28 & 2.63 & 3.22 \\
\hline & 4 & 2.65 & 3.23 & 3.86 & 4.28 & 2.63 & 3.22 \\
\hline & 5 & 2.65 & 3.23 & 3.86 & 4.28 & 2.63 & 3.22 \\
\hline & 6 & 7.31 & 0.00 & 33.57 & 29.83 & 7.02 & 0.00 \\
\hline 16 & 1 & 7.21 & 0.00 & 28.20 & 25.21 & 15.00 & 13.86 \\
\hline 18 & 1 & 80.32 & 70.03 & 8.99 & 0.00 & 102.15 & 88.81 \\
\hline 21 & 1 & 19.00 & 0.00 & 48.96 & 43.06 & 19.18 & 0.00 \\
\hline \multirow{6}{*}{22} & 1 & 1.57 & 0.00 & 0.04 & 0.00 & 1.54 & 0.00 \\
\hline & 2 & 1.57 & 0.00 & 0.04 & 0.00 & 1.54 & 0.00 \\
\hline & 3 & 1.57 & 0.00 & 0.04 & 0.00 & 1.54 & 0.00 \\
\hline & 4 & 1.57 & 0.00 & 0.04 & 0.00 & 1.54 & 0.00 \\
\hline & 5 & 1.57 & 0.00 & 0.04 & 0.00 & 1.54 & 0.00 \\
\hline & 6 & 1.57 & 0.00 & 0.04 & 0.00 & 1.54 & 0.00 \\
\hline \multirow{3}{*}{23} & 1 & 7.26 & 0.00 & 2.10 & 0.00 & 7.98 & 0.00 \\
\hline & 2 & 7.26 & 0.00 & 2.10 & 0.00 & 7.98 & 0.00 \\
\hline & 3 & 52.08 & 45.75 & 45.14 & 39.78 & 53.05 & 46.59 \\
\hline \multicolumn{2}{|c|}{ Total } & 496.77 & 385.99 & $\begin{array}{l}573.24 \\
\end{array}$ & 554.96 & $\begin{array}{l}590.35 \\
\end{array}$ & 506.37 \\
\hline
\end{tabular}

${ }^{\text {a }}$ Synchronous condenser (SC)

The system output control variables such as generator bus voltages, transformer tap settings and shunt inductor obtained after optimization in all three cases are given in Table 4. The generators reactive power output schedules and their respective payments obtained from all three test cases are compared in Table 5. It is observed that the total payment for reactive power support services from generators and synchronous condensers is achieved of extreme values i.e. maximum in case 2 and minimum in case 1, which are the cases of single objective optimization framework (see 
Table 5). The TPF for reactive power supports is of moderate value obtained from proposed MORPMC model as in cases 3.

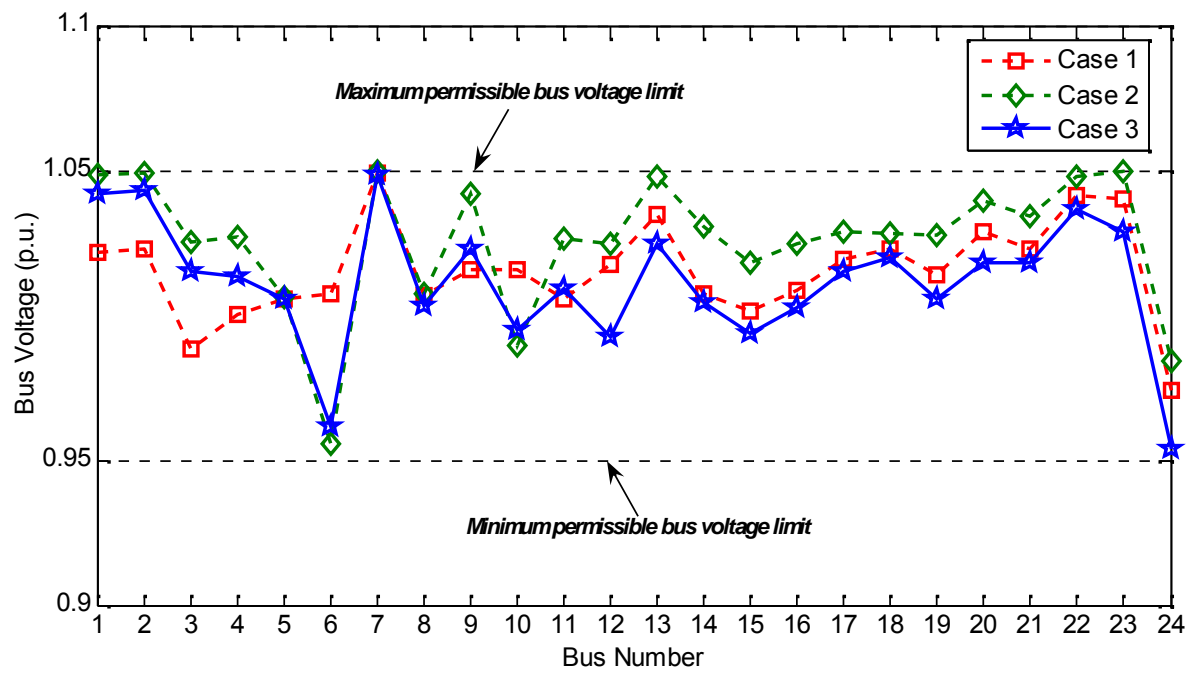

Figure 5. Comparison of bus voltage profiles obtained after optimization for RPMC models

The reactive power generation output schedule obtained from proposed NSGA-II based MORPMC model (best compromised solution) is graphically shown as in Figure 4. It is clear that there is no violation in generators reactive power outputs. In other words, all the generators reactive power output values are within their corresponding ranges of minimum and maximum permissible limits in case 4 . The bus voltage profiles obtained from all three cases are also compared as shown in Figure 5.

\section{CONCLUSION}

In this work, the reactive power market clearing problem is formulated as complex multiobjective optimization problem, in which two objective functions such as TPF and VSEI are optimized simultaneously while satisfying different system equality and inequality constraints using NSGA-II based multi-objective optimization technique. The MO-RPMC model is tested on standard IEEE-24 bus RTS and its optimization results are compared with single-objective optimization results. It is concluded that the NSGA-II based RPMC provides superior results as compared to the same obtained from RCGA based single-objective RPMC models and helps to take better market clearing decisions by ISO in competitive environment.

\section{REFERENCES}

[1] Deb K., (2001) Multi-objective optimization using evolutionary algorithms, New York: John Wiley and Sons, Inc.

[2] Abido M A \& Bakhashwain J M, (2005) "Optimal VAR dispatch using a multi-objective evolutionary algorithm," Int. J Electrical Power \& Energy Systems, Vol. 27, Vol. 1, pp. 13-20.

[3] Zhang W \& Liu Y, (2008) "Multi-objective reactive power and voltage control based on fuzzy optimization strategy and fuzzy adaptive particle swarm," Int. J Electrical Power \& Energy Systems, Vol. 30, pp. 525-532.

[4] Dai C, Chen W, Zhu Y \& Zhang X., (2009) "Reactive power dispatch considering voltage stability with seekers optimization algorithm," Electric Power System Research, Vol. 79, pp. 1462-1471. 
[5] Jeyadevi S, Baskar S, Babulal C K \& Iruthayarajan M W, (2011) "Solving multiobjective optimal reactive power dispatch using modified NSGA-II," Int. J Electrical Power \& Energy Systems, Vol. 33, pp. 219-228.

[6] Esmaili M, Shayanfar H A \& Amjady N, (2009) "Multi-objective congestion management incorporating voltage and transient stabilities," Energy, Vol. 34, pp. 1401-1412.

[7] Rabiee A, Shayanfar H A \& Amjady N, "Multiobjective clearing of reactive power market considering power system security," Applied Energy, Vol. 86, No. 9, pp. 1555-1564.

[8] Hao S, \& Papalexopoulos A, (1997) "Reactive power pricing and management," IEEE Transactions on Power Systems, Vol. 12, No. 1, pp. 95-104.

[9] Wang Y \& Xu W, (2004) "An investigation on the reactive power support service need of power producers," IEEE Transactions on Power Systems, Vol. 19, No. 1, pp. 586-593.

[10]Hao S, (2003) “A Reactive Power Management Proposal for Transmission Operators," IEEE Transactions on Power Systems, Vol. 18, No. 4, pp. 1374-1381.

[11] Bhattacharya K \& Zhong J, (2001) "Reactive Power as an Ancillary Service," IEEE Transactions on Power Systems, Vol. 16, No. 2, pp. 294-300.

[12]Zhong J \& Bhattacharya K, (2002) "Toward a Competitive Market for Reactive Power," IEEE Transactions on Power Systems, Vol. 17, No. 4, pp. 1206-1215.

[13] Kessel P \& Glavitsch H, (1986) "Estimating the voltage stability of power systems," IEEE Transactions on Power Systems, Vol. 1, pp. 346-354.

[14] Vyjayanthi C \& Thukaram D, (2011) "Evaluation and improvement of generators reactive power margins in interconnected power systems," IET Generation Transmission \& Distribution, Vol. 5, Issue 4, pp. 504-518.

[15] Deb K, Pratap A, Agarwal S \& Meyarivan T, (2002) "A Fast Elitist Multi-objective Genetic Algorithm: NSGA-II,” IEEE Transactions on Evolutionary Computations, Vol. 6, No. 2, pp. 182197.

[16] Saraswat A \& Saini A, (2011) "Optimal Reactive Power Dispatch by an Improved Real Coded Genetic Algorithm with PCA Mutation” Proc. IET, Second International Conference on Sustainable Energy and Intelligent System (IET SEISCON 2011), Vol. 2, pp. 310-315.

[17] Dhillon J S, Parti S C \& Kothari D P, (1993) "Stochastic economic emission load dispatch," Electric Power System Research, Vol. 26, pp. 179-186.

[18] Reliability Test System Task Force, (1999) The IEEE reliability test system - 1996. IEEE Transactions on Power Systems, Vol. 14, No. 3, pp. 1010-1020.

[19] Rabiee A, Shayanfar H \& Amjady N, (2009) "Coupled energy and reactive power market clearing considering power system security,” Energy Conversion and Management, Vol. 50, pp. 907-915.

\section{Authors}

Ashish Saini, Ph.D., is presently working as an Associate Professor in the Dept. of Electrical Engg., Faculty of Engineering, Dayalbagh Educational Institute, Agra, India. His research interest includes the applications of artificial intelligence techniques in power system optimization, planning and operation of power systems, power system deregulation and congestion management.

Amit Saraswat received his B.Sc.Engg. (Electrical Engg.) and M.Tech. (Engineering Systems) from Faculty of Engineering, Dayalbagh Educational Institute, Agra, India in 2003 and 2006 respectively. Presently, he is pursuing his Ph.D. (Power Systems) from the same institution. His research area includes competitive electricity markets, reactive power management, applications of soft computing techniques in power systems etc.

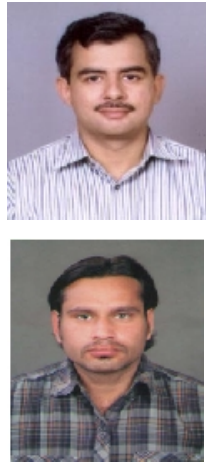

